\title{
Integrated Analysis of the Endoscopic, Pathological and Molecular Characteristics of Colorectal Tumorigenesis
}

\author{
Hiromu Suzukia Eiichiro Yamamoto ${ }^{a, b}$ Hiro-o Yamano ${ }^{b}$ Hiroshi Nakase $^{b}$ \\ Tamotsu Sugaic

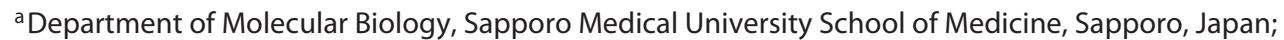 \\ ${ }^{b}$ Department of Gastroenterology and Hepatology, Sapporo Medical University School of Medicine, Sapporo, Japan; \\ 'Department of Molecular Diagnostic Pathology, School of Medicine, Iwate Medical University, Morioka, Japan
}

\section{Keywords}

Colorectal cancer · Serrated lesion · Pit pattern · DNA methylation - Secreted protein acidic and rich in cysteinerelated modular calcium binding 1 - Laterally spreading tumor · Neurotensin receptor 1

\begin{abstract}
Background: Colorectal cancers (CRCs) develop through the accumulation of genetic and epigenetic alterations of oncogenes and tumor suppressor genes. In addition to the wellcharacterized adenoma-carcinoma sequence, the serrated neoplasia pathway is now recognized as an alternative pathway for CRC development. Summary: Through analysis of the colonoscopic, pathological, and molecular features of colorectal tumors, we identified a novel microsurface structure characteristic of serrated lesions. The Type II-Open (Type II-O) pit pattern is highly specific to sessile serrated adeno$\mathrm{ma} /$ polyps (SSA/Ps), and Type-II-O-positive tumors frequently exhibit v-raf murine sarcoma viral oncogene homolog B1 (BRAF) mutation and 5'-C-phosphate-G-3' (CpG) island hypermethylation. By screening DNA methylation associated with the development of serrated lesions, we detected methylation of secreted protein acidic and rich in cysteine (SPARC)-related modular calcium binding 1 (SMOC1) in tra-
\end{abstract}

\section{KARGER}

(c) 2018 S. Karger AG, Basel

E-Mail karger@karger.com

www.karger.com/dig ditional serrated adenomas (TSAs). Epigenetic silencing of SMOC1 is prevalent among TSAs but it is rarely observed in SSA/Ps, which suggests SMOC1 could be a useful diagnostic marker of serrated lesions. We also searched for epigenetic alterations associated with the growth pattern of colorectal tumors and found that methylation of neurotensin receptor 1 is associated with lateral and non-invasive tumor growth. Key Message: Through the summarized studies, we have been able to identify novel morphological and molecular features that could contribute to a better understanding of colorectal tumors and to improved clinical diagnosis.

(c) 2018 S. Karger AG, Basel

\section{Introduction}

Colorectal cancer (CRC) is a leading cause of cancer mortality worldwide. CRCs develop through the accumulation of multiple genetic and epigenetic alterations of oncogenes and tumor suppressor genes. In the wellcharacterized adenoma-carcinoma sequence model, CRCs arise from adenomas through the accumulation of genetic alterations of key genes that include APC, v-Kiras2 kirsten rat sarcoma viral oncogene homolog (KRAS), and tumor protein p53 (TP53) (Fig. 1) [1]. In 
addition, advances in cancer genome biology revealed that CRCs can be categorized into several subgroups based on their molecular characteristics. For instance, CRCs are broadly divided into 2 groups based on whether they exhibit chromosomal instability or microsatellite instability (MSI) [2]. From an epigenetic viewpoint, moreover, a subset of CRC exhibit concurrent hypermethylation of multiple $\mathrm{CpG}$ islands, which is referred to as the 5'-C-phosphate-G-3' (CpG) island methylator phenotype (CIMP) [3]. CRCs with CIMP exhibit several characteristic features, including frequent $\mathrm{v}$-raf $\mathrm{mu}$ rine sarcoma viral oncogene homolog B1 (BRAF) mutation, infrequent TP53 mutation, loss of mutL homolog 1 (MLH1) expression due to CpG island hypermethylation, and resultant MSI [3].

In addition to the adenoma-carcinoma sequence, the serrated neoplasia pathway is now recognized as an alternative pathway toward CRC development [4]. Serrated lesions are subcategorized into hyperplastic polyps (HPs), sessile serrated adenoma/polyps (SSA/Ps), and traditional serrated adenomas (TSAs) [4]. In the past, serrated lesions were simply classified as HPs and considered to be without malignant potential. However, evidence now strongly indicates that SSA/Ps and TSAs are important premalignant lesions. In particular, because SSA/Ps frequently exhibit BRAF mutation and hypermethylation of multiple CpG islands, SSA/Ps are thought to be precursors of CRCs with MSI (Fig. 1) [4]. To understand the molecular basis of colorectal tumorigenesis and apply that knowledge to achieve better diagnosis and treatment of CRC, we carried out an integrative analysis of the colonoscopic, pathological and molecular characteristics of premalignant and malignant colorectal lesions. In this review, we describe correlations between the morphological features and molecular alterations in colorectal lesions as well as novel epigenetic events potentially associated with tumor development.

\section{Associations between Microsurface Structures and Molecular Alterations in Serrated Lesions}

High-resolution magnifying colonoscopy is a powerful diagnostic tool for evaluating the malignant potential of colorectal lesions [5, 6]. Using Kudo's classification, the pit patterns of non-neoplastic lesions are classified as Type I (normal) or Type II (HP), while the pit patterns of neoplastic lesions are classified as Types III, IV, and V, among which Type $\mathrm{V}$ lesions are considered to be cancers [5]. When this pit pattern classification was first estab-



Serrated neoplasia pathway

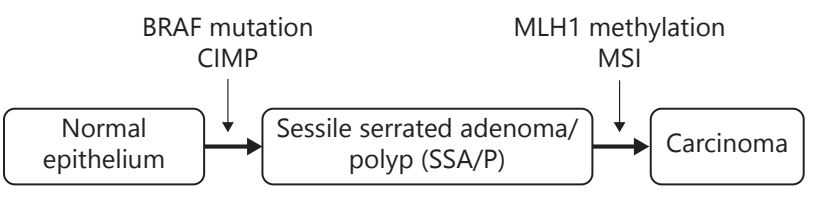

Fig. 1. Models of CRC development. Shown are the adenoma-carcinoma sequence and the serrated neoplasia pathway.

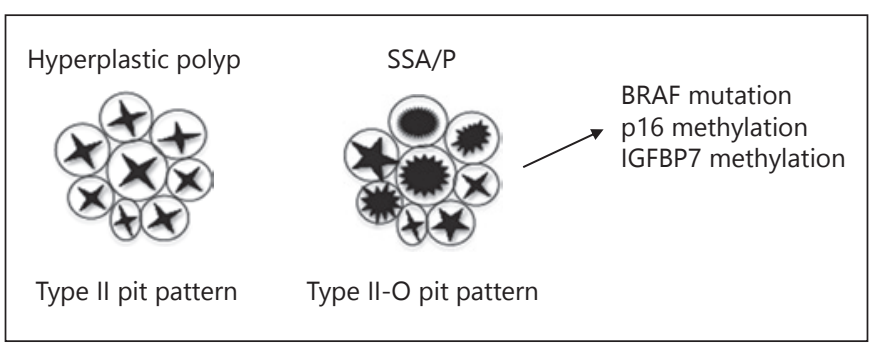

Fig. 2. Microsurface structures (pit patterns) of hyperplastic polyps and SSA/Ps. The Type II-O pit pattern is a characteristic feature of SSA/Ps, and Type II-O-positive tumors frequently show BRAF mutation and hypermethylation of multiple genes, including p16 and IGFBP7.

lished, SSA/Ps and TSAs were considered to be non-neoplastic lesions because they exhibit the Type II pit pattern. However, we hypothesized that serrated lesions with neoplastic potential may present specific morphological features that are distinct from non-neoplastic lesions. By comparing the morphological features of a series of serrated lesions and conventional adenomas, we found that SSA/Ps possess a characteristic pit pattern that is similar to the conventional Type II, but the pits were wider and more rounded in shape, reflecting crypt dilation [7]. We termed this pattern Type II-Open (Type II-O) and further analyzed its clinical and biological significance (Fig. 2).

The Type II-O pattern was highly specific to SSA/Ps, and a majority of Type II-O-positive lesions exhibited BRAF mutation and CIMP, while they infrequently showed KRAS mutation [7]. These results suggest that the Type II-O pit pattern may be a hallmark of lesions with the potential to develop into CIMP-positive/MSI CRCs. 
To test this idea, we analyzed lesions in which portions exhibiting Type II-O patterns were present along with portions exhibiting more advanced pit patterns (Type III, IV or V). Both subcomponents exhibited similar molecular features (BRAF mutation and CIMP), suggesting they arose from a single origin, though subcomponents with advanced pit patterns showed higher levels of p16 and MLH1 methylation.

In a subsequent study, we further investigated the clinical implications of the Type II-O pit pattern. SSA/Ps with cytological dysplasia are reportedly characterized by frequent MLH1 methylation and are at a high risk of developing into CRC [8]. We found that the Type II-O plus Type III/IV pit pattern is a common feature of SSA/Ps with cytological dysplasia in both the proximal and distal colon [9], which suggests that progression of SSA/Ps to more advanced lesions is associated with further accumulation of molecular alterations and morphological changes.

We also explored the microsurface structures characteristic of TSAs and recently reported that Type II pit patterns could be subcategorized into classical Type II, Type II-O, and Type II-Long (Type II-L) [10]. Most lesions with simple Type II or Type II-L patterns were HPs, while mixtures of Type II or Type II-L plus Type III/IV patterns were characteristic of TSAs. We therefore propose that Type II-L-positive TSAs may develop into KRAS-mutated/CIMP-low/microsatellite stable CRCs, although further study will be necessary to confirm this hypothesis.

\section{Epigenetic Silencing of Secreted Protein Acidic and Rich in Cysteine-Related Modular Calcium Binding 1 in TSAs}

In contrast to SSA/Ps, TSAs are thought to develop into microsatellite stable CRCs, though the underlying mechanism of tumor progression is not yet fully understood [11]. TSAs reportedly exhibit genetic and epigenetic characteristics distinct from those of SSA/Ps. For instance, PTPRK-RSPO3 fusion and RNF43 mutations are reported to be characteristic features of TSAs $[12,13]$. TSAs mostly harbor KRAS or BRAF mutations, and those with BRAF mutations have features similar to SSA/Ps, such as a proximal colon location and CIMP-high [11, 14]. However, TSAs rarely show MLH1 methylation. CIMP-high is infrequently found in KRAS mutant and BRAF/KRAS wild-type TSAs, while approximately half of these lesions show CIMP-low [11]. These results indicate that aberrant DNA methylation likely plays an important role in the development of TSAs.

Endoscopic, Pathological and Molecular Characteristics of Colorectal Tumorigenesis
To identify DNA methylation associated with the TSA development, we analyzed a series of TSAs containing both protruding and flat components within the same tumors [15] (Fig. 3). As the flat components are considered precursors of the protruding portions, we compared the genome wide DNA methylation status between the 2 components. We identified 11 genes (B3GALNT1, CADPS, FAM92A1, FEZ1, FRMD4B, GABRA4, KIAA1529, OGFRL1, PRDM16, SMOC1, and ZNF34) in which methylation levels were elevated in the protruding components. Among them, we noted that secreted protein acidic and rich in cysteine (SPARC)-related modular calcium binding 1 (SMOC1) was frequently methylated in TSAs but was rarely methylated in SSA/Ps. SMOC1 belongs to the SPARC family of matricellular proteins, which has 8 known members: SPARC, SPARCL1/Hevin, SPOCK1, -2, -3, SMOC1, -2 and FSTL1. Although the biological function of SMOC1 is not fully understood, it is reportedly associated with osteoblast differentiation, ocular and limb development, and angiogenesis [15-17]. Members of SPARC family have been implicated in various tumor types. For instance, SPARC is silenced by DNA methylation in CRC and it may act as a tumor suppressor $[18,19]$. SMOC1 is known to be overexpressed in brain tumors and methylated in breast cancer, but its role in tumorigenesis remains largely unknown $[20,21]$.

Our immunohistochemical analysis revealed that SMOC1 expression is reduced in TSAs, whereas it is abundantly expressed in normal colon and SSA/Ps [15]. This suggests that SMOC1 could be a useful marker for discriminating SSA/Ps from TSAs. Moreover, lesions in which TSA and cancerous components were present together showed higher levels of SMOC1 methylation than TSAs without cancer, indicating that SMOC1 methylation may be associated with the risk of CRC development.

When we further analyzed SMOC1 methylation in a large number of clinical specimens, we found that SMOC1 was also frequently methylated in conventional adenomas and CRCs [15]. For non-invasive tumors, SMOC1 methylation was positively associated with older age, larger tumor size, KRAS mutation, TP53 mutation, and CIMP-low. In invasive tumors, SMOC1 methylation was again associated with KRAS mutation and CIMP-low. Analysis using CRC cell lines showed that SMOC1 methylation is associated with gene silencing, and ectopic expression of SMOC1 suppressed CRC cell proliferation and xenograft formation in nude mice, which is indicative of its tumor suppressor function. Collectively, these results suggest that SMOC1 methylation may play a key role in the development of TSAs and adenomas that could progress to CIMP-low CRCs [15]. 
Fig. 3. Identification of SMOC1 methylation in TSA. a To identify molecular alterations associated with TSA development, biopsy specimens were collected from flat and protruding components of TSAs, after which the molecular alterations were analyzed. b DNA methylation profiles were compared between the flat and protruding components of TSAs. CpG sites differentially methylated between the 2 components were selected, and SMOC1 methylation was identified.

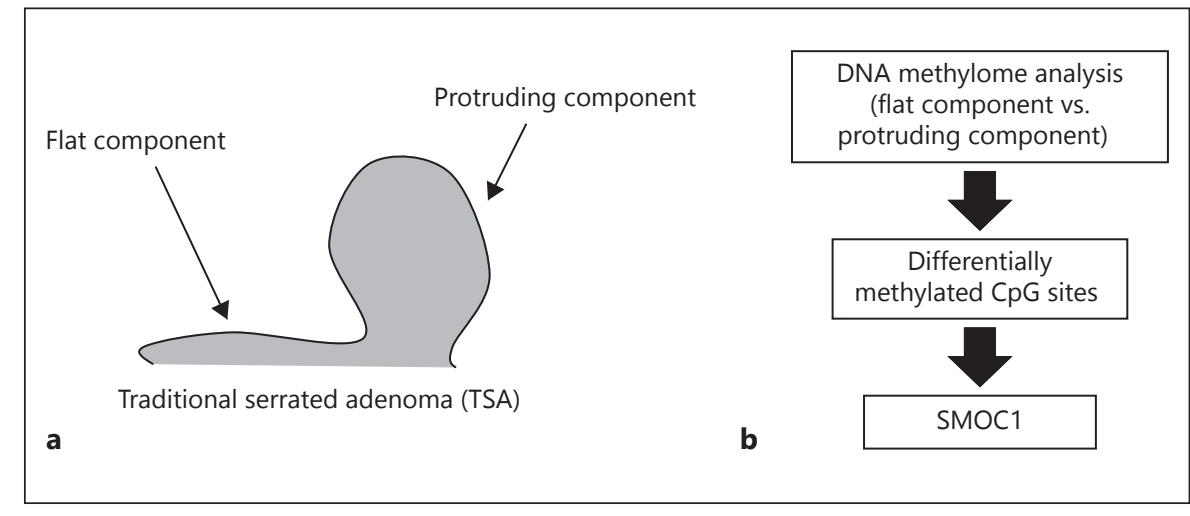

Fig. 4. A screen for genes associated with the growth patterns of colorectal tumors. a By comparing DNA methylation profiles between large/non-invasive and small/invasive tumors, frequent NTSR1 methylation in large/non-invasive tumors was identified. b NTS-NTSR1 signaling promotes tumor cell survival, proliferation, migration, and invasion.



\section{Epigenetic Silencing of Neurotensin Receptor 1 is Associated with Lateral and Non-Invasive Tumor Growth}

In contrast to the classical adenoma-carcinoma sequence, a subset of CRCs develop from non-polypoid lesions. Laterally spreading tumors (LSTs) are tumors that extend laterally along the luminal wall and are greater than $10 \mathrm{~mm}$ in diameter with a low vertical axis [22]. LSTs are categorized into 2 subtypes: those with a granular morphology (LST-G) and those with a flat or non-granular morphology (LST-NG) [23, 24]. Although the molecular mechanism involved in the development of LSTs is not fully understood, several studies reported frequent detection of KRAS mutation and CIMP in LSTs $[25,26]$. A more recent study showed that LST-G is associated with KRAS mutation and the intermediate-methylation epigenotype, whileLST-NG ischaracterized by catenin beta-1 (CTNNB1) mutation and a low-methylation epigenotype [27].

The above findings suggest that aberrant DNA methylation may be an important determinant of the lateral or vertical growth pattern of colorectal tumors. We therefore compared the DNA methylation profiles of large ( $\geq 20 \mathrm{~mm}$ in diameter) and non-invasive tumors with those in small ( $<20 \mathrm{~mm}$ in diameter) and invasive tumors (Fig. 4) [28]. We found that a substantial number of genes were differentially methylated among these tumor types and identified neurotensin receptor 1 (NTSR1) as a gene that is preferentially methylated in large, non-invasive tumors. Neurotensin (NTS) is a 13-amino acid neuropeptide localized mainly in the central nervous system and the distal small bowel [29]. The physiological functions of NTS include modulation of gastrointestinal tract motility, stimulation of intestinal secretion, and promotion of the growth and regeneration of intestinal epithelial cells [29]. Activation of NTS-NTSR1 signaling has been implicated in the progression of various cancers, including CRC [30].

Among non-invasive colorectal tumors, levels of NTSR1 methylation were significantly higher in LST-G than other types of tumors [28]. We also noted that higher levels of NTSR1 methylation were associated with bet- 
ter overall survival of patients with invasive colorectal tumors. Analysis using CRC cell lines showed that NTSR1 methylation is associated with its transcriptional silencing, and that ectopic expression of NTSR1 promoted CRC cell proliferation and invasion, which is consistent with the known oncogenic function of NTS-NTSR1 signaling (Fig. 4) [30].

The results summarized above suggest that despite functioning as an oncogene, NTSR1 is a target of epigenetic silencing in colorectal tumors. To investigate the biological significance of NTSR1 methylation in colorectal tumorigenesis, we examined mixed colorectal lesions possessing adenomatous and malignant components. We found that NTSR1 was methylated and silenced in the adenomatous regions but was unmethylated and abundantly expressed in cancerous regions [28]. This suggests that NTSR1 is a methylation-prone gene early during tumorigenesis, but its activation is necessary for malignant progression. It remains unclear, however, whether tumor cells actively demethylate NTSR1 or whether there is selective survival of tumor cells without NTSR1 methylation during malignant progression. These results suggest that NTSR1 methylation may be associated with the lateral and non-invasive growth of colorectal tumors and could be a prognostic marker of CRC.

\section{Conclusions}

In a series of studies, we have shown that microsurface structures are associated with molecular alterations and progression of serrate lesions. As colonoscopic identification and resection of lesions with malignant potential is an effective strategy for CRC prevention, our findings could contribute to better identification and treatment of premalignant colorectal lesions. In addition, through performance of an integrated analysis of endoscopic, pathologi$\mathrm{cal}$, and molecular characteristics, we identified novel epigenetic alterations potentially associated with the development of colorectal tumors. Although further study will be necessary to fully understand the roles of these genes in tumorigenesis, they appear to have the potential to serve as diagnostic or predictive markers of colorectal tumors.

\section{Acknowledgements}

We thank Dr. William F. Goldman for editing the manuscript.

\section{Disclosure Statement}

All authors have no conflict of interest to declare.

\section{References}

1 Fearon ER, Vogelstein B: A genetic model for colorectal tumorigenesis. Cell 1990;61:759767.

2 Carethers JM, Jung BH: Genetics and genetic biomarkers in sporadic colorectal cancer. Gastroenterology 2015;149:1177-1190.e3.

3 Suzuki H, Yamamoto E, Maruyama R, Niinuma T, Kai M: Biological significance of the $\mathrm{CpG}$ island methylator phenotype. Biochem Biophys Res Commun 2014;455:35-42.

4 IJspeert JE, Vermeulen L, Meijer GA, Dekker E: Serrated neoplasia-role in colorectal and clinical implications. Nat Rev Gastroenterol Hepatol 2015;12:401-409.

5 Kudo S, Tamura S, Nakajima T, Yamano H, Kusaka H, Watanabe H: Diagnosis of colorectal tumorous lesions by magnifying endoscopy. Gastrointest Endosc 1996;44:8-14.

6 Sanomura M, Tanaka S, Sasaki Y, Fukunishi $\mathrm{S}$, Higuchi K: Endoscopic diagnosis of the invasion depth of T1 colorectal carcinoma for endoscopic resection by using narrow-band imaging magnification as total excisional biopsy. Digestion 2016;94:106-113.

7 Kimura T, Yamamoto E, Yamano HO, Suzuki H, Kamimae S, Nojima M, Sawada T, Ashida M, Yoshikawa K, Takagi R, Kato R, Harada T,
Suzuki R, Maruyama R, Kai M, Imai K, Shinomura Y, Sugai T, Toyota M: A novel pit pattern identifies the precursor of colorectal cancer derived from sessile serrated adenoma. Am J Gastroenterol 2012;107:460-469.

8 Burgess NG, Pellise M, Nanda KS, Hourigan LF, Zanati SA, Brown GJ, Singh R, Williams SJ, Raftopoulos SC, Ormonde D, Moss A, Byth K, P’Ng H, McLeod D, Bourke MJ: Clinical and endoscopic predictors of cytological dysplasia or cancer in a prospective multicentre study of large sessile serrated adenomas/ polyps. Gut 2016;65:437-446.

9 Tanaka Y, Yamano HO, Yamamoto E, Matushita HO, Aoki H, Yoshikawa K, Takagi R, Harada E, Nakaoka M, Yoshida Y, Eizuka M, Sugai T, Suzuki H, Nakase H: Endoscopic and molecular characterization of colorectal sessile serrated adenoma/polyps with cytologic dysplasia. Gastrointest Endosc 2017;86:11311138.e4.

10 Aoki H, Yamamoto E, Yamano HO, Sugai T, Kimura T, Tanaka Y, Matsushita HO, Yoshikawa K, Takagi R, Harada E, Nakaoka M, Yoshida Y, Harada T, Sudo G, Eizuka M, Yorozu A, Kitajima H, Niinuma T, Kai M, Nojima M, Suzuki H, Nakase H: Subtypes of the Type II pit pattern reflect distinct molecular subclasses in the serrated neoplastic pathway. Dig Dis Sci 2018;63:1920-1928.

11 Bettington ML, Walker NI, Rosty C, Brown IS, Clouston AD, McKeone DM, Pearson SA, Klein K, Leggett BA, Whitehall VL: A clinicopathological and molecular analysis of 200 traditional serrated adenomas. Mod Pathol 2015;28:414-427.

12 Sekine S, Yamashita S, Tanabe T, Hashimoto T, Yoshida H, Taniguchi H, Kojima M, Shinmura K, Saito Y, Hiraoka N, Ushijima T, Ochiai A: Frequent PTPRK-RSPO3 fusions and RNF43 mutations in colorectal traditional serrated adenoma. J Pathol 2016;239:133-138.

13 Tsai JH, Liau JY, Yuan CT, Lin YL, Tseng LH, Cheng ML, Jeng YM: RNF43 is an early and specific mutated gene in the serrated pathway, with increased frequency in traditional serrated adenoma and its associated malignancy. Am J Surg Pathol 2016;40:1352-1359.

14 Wiland HO 4th, Shadrach B, Allende D, Carver P, Goldblum JR, Liu X, Patil DT, Rybicki LA, Pai RK: Morphologic and molecular characterization of traditional serrated adenomas of the distal colon and rectum. Am J Surg Pathol 2014;38:1290-1297.
Endoscopic, Pathological and Molecular Characteristics of Colorectal Tumorigenesis
Digestion 2019;99:33-38 DOI: $10.1159 / 000494410$ 
15 Aoki H, Yamamoto E, Takasawa A, Niinuma T, Yamano HO, Harada T, Matsushita HO, Yoshikawa K, Takagi R, Harada E, Tanaka Y, Yoshida Y, Aoyama T, Eizuka M, Yorozu A, Kitajima H, Kai M, Sawada N, Sugai T, Nakase H, Suzuki H: Epigenetic silencing of SMOC1 in traditional serrated adenoma and colorectal cancer. Oncotarget 2018;9:47074721.

16 Choi YA, Lim J, Kim KM, Acharya B, Cho JY, Bae YC, Shin HI, Kim SY, Park EK: Secretome analysis of human BMSCs and identification of SMOC1 as an important ECM protein in osteoblast differentiation. J Proteome Res 2010;9:2946-2956.

17 Okada I, Hamanoue H, Terada K, Tohma T, Megarbane A, Chouery E, Abou-Ghoch J, Jalkh N, Cogulu O, Ozkinay F, Horie K, Takeda J, Furuichi T, Ikegawa S, Nishiyama K, Miyatake S, Nishimura A, Mizuguchi T, Niikawa N, Hirahara F, Kaname T, Yoshiura K, Tsurusaki Y, Doi H, Miyake N, Furukawa T, Matsumoto N, Saitsu H: SMOC1 is essential for ocular and limb development in humans and mice. Am J Hum Genet 2011;88: $30-41$.

18 Cheetham S, Tang MJ, Mesak F, Kennecke H, Owen D, Tai IT: SPARC promoter hypermethylation in colorectal cancers can be reversed by 5 -Aza-2'deoxycytidine to increase SPARC expression and improve therapy response. Br J Cancer 2008;98:1810-1819.

19 Aoi W, Naito Y, Takagi T, Tanimura Y, Takanami Y, Kawai Y, Sakuma K, Hang LP, Mizushima K, Hirai Y, Koyama R, Wada S,
Higashi A, Kokura S, Ichikawa H, Yoshikawa $\mathrm{T}$ : A novel myokine, secreted protein acidic and rich in cysteine (SPARC), suppresses colon tumorigenesis via regular exercise. Gut 2013;62:882-889.

20 Brellier F, Ruggiero S, Zwolanek D, Martina E, Hess D, Brown-Luedi M, Hartmann U, Koch M, Merlo A, Lino M, Chiquet-Ehrismann R: SMOC1 is a tenascin-C interacting protein over-expressed in brain tumors. Matrix Biol 2011;30:225-233.

21 Fackler MJ, Umbricht CB, Williams D, Argani P, Cruz LA, Merino VF, Teo WW, Zhang Z, Huang P, Visvananthan K, Marks J, Ethier S, Gray JW, Wolff AC, Cope LM, Sukumar S: Genome-wide methylation analysis identifies genes specific to breast cancer hormone receptor status and risk of recurrence. Cancer Res 2011;71:6195-6207.

22 Kudo S: Endoscopic mucosal resection of flat and depressed types of early colorectal cancer. Endoscopy 1993;25:455-461.

23 Igawa A, Oka S, Tanaka S, Otani I, Kunihara S, Chayama K: Evaluation for the clinical efficacy of colon capsule endoscopy in the detection of laterally spreading tumors. Digestion 2017;95:43-48.

24 Hurlstone DP, Cross SS, Adam I, Shorthouse AJ, Brown S, Sanders DS, Lobo AJ: Efficacy of high magnification chromoscopic colonoscopy for the diagnosis of neoplasia in flat and depressed lesions of the colorectum: a prospective analysis. Gut 2004;53:284-290.

25 Hiraoka S, Kato J, Tatsukawa M, Harada K, Fujita H, Morikawa T, Shiraha H, Shiratori Y:
Laterally spreading type of colorectal adenoma exhibits a unique methylation phenotype and K-ras mutations. Gastroenterology 2006; 131:379-389.

26 Kaji E, Kato J, Suzuki H, Akita M, Horii J, Saito S, Higashi R, Ishikawa S, Kuriyama M, Hiraoka S, Uraoka T, Yamamoto K: Analysis of $\mathrm{K}$-ras, BRAF, and PIK3CA mutations in laterally-spreading tumors of the colorectum. J Gastroenterol Hepatol 2011;26:599607.

27 Sakai E, Ohata K, Chiba H, Matsuhashi N, Doi $\mathrm{N}$, Fukushima J, Endo H, Takahashi H, Tsuji S, Yagi K, Matsusaka K, Aburatani H, Nakajima A, Kaneda A: Methylation epigenotypes and genetic features in colorectal laterally spreading tumors. Int J Cancer 2014;135: 1586-1595

28 Kamimae S, Yamamoto E, Kai M, Niinuma T, Yamano HO, Nojima M, Yoshikawa K, Kimura T, Takagi R, Harada E, Harada T, Maruyama R, Sasaki Y, Tokino T, Shinomura Y, Sugai T, Imai K, Suzuki H: Epigenetic silencing of NTSR1 is associated with lateral and noninvasive growth of colorectal tumors. Oncotarget 2015;6:29975-29990.

29 Thomas RP, Hellmich MR, Townsend CM Jr, Evers BM: Role of gastrointestinal hormones in the proliferation of normal and neoplastic tissues. Endocr Rev 2003;24:571-599.

$30 \mathrm{Wu}$ Z, Martinez-Fong D, Tredaniel J, Forgez $P$ : Neurotensin and its high affinity receptor 1 as a potential pharmacological target in cancer therapy. Front Endocrinol (Lausanne) 2012;3:184 